\title{
GENERALISED NUCLEAR MAPS IN NORMED LINEAR SPACES
}

\author{
BY M. S. RAMANUJAN
}

Communicated by F. W. Gehring, January 12, 1970

1. Preliminary definitions and notations. Grothendieck [3] and Pietsch [6] present an exhaustive study of nuclear operators and nuclear maps. The notion of a nuclear operator was extended by Persson and Pietsch in a recent paper [5] and they study in detail the $p$-nuclear and quasi-p-nuclear maps. In this paper we define and study certain linear maps called $\lambda$-nuclear and quasi- $\lambda$-nuclear maps. Our definition and generalisation here are motivated by the Köthe sequence spaces and their duality theory. For the special case $\lambda=l^{1}$ we obtain the nuclear operators and for $\lambda=l^{p}$ we obtain the $p$-nuclear maps; also, the special case $\lambda=c_{0}$ yields the $\infty$-nuclear operators of Persson and Pietsch. Most of the results in this work are motivated by the work of Persson and Pietsch [5] and Köthe sequence spaces.

We shall briefly outline our assumptions. For definitions not stated here see Garling [1], Köthe [4], Ruckle [7], Sargent [9] and Zeller [10]. Let $\lambda$ be a symmetric sequence space of scalars and $\lambda^{*}$ be its Köthe dual. We shall assume that $\lambda$ is provided with the Mackey topology of the duality $\left\langle\lambda, \lambda^{*}\right\rangle$ and that this topology is provided by a norm $p, p$ itself being an extended seminorm on $\omega$. We assume now that $\lambda$ is solid and that it is $K$-symmetric, i.e., for each $x \in \lambda$ and for each permutation $\pi$ of $I^{+}$we have $x_{\pi} \in \lambda$ and $p(x)=p\left(x_{\pi}\right) . \lambda$ is also assumed to be a BK space with AK. We remark that our assumptions imply that $\lambda=\omega$ or $\lambda=l^{\infty}$ or $\lambda \subseteq c_{0}$. The space $\lambda^{*}$ is now considered as the topological dual of $\lambda$ and equipped with its natural norm topology.

We pause now to point out that in addition to the spaces $l^{p}, 1 \leqq p$ $<\infty$, the sequence spaces $n(\phi)$ of Sargent [8] and the sequence spaces $\mu_{a, p}$ and $\nu_{a, p}$ of Garling [2] serve as examples of the type of sequence spaces $\lambda$ we consider. Garling shows also that his spaces $\mu_{a, p}$ are in general not linearly homeomorphic to $l^{p}$.

Next let $E$ and $F$ be normed linear spaces. Then $\lambda(E)$ is the (vector sequence) space of all vectors $x=\left(x_{n}\right), x_{n} \in E$ for each $n$ and such that the sequence $\left(\left\langle x_{n}, a\right\rangle\right) \in \lambda$ for each $a \in E^{\prime}$. Formally define

$$
\epsilon_{\lambda}(x)=\sup _{\|a\| \leqq 1} p\left(\left|\left\langle x_{n}, a\right\rangle\right|\right),
$$

where $p$ is the norm on $\lambda$.

AMS Subject Classifications. Primary 4710.

Key Words and Phrases. Generalised nuclear maps. 
$\lambda[E]$ is the space of sequences $x=\left(x_{n}\right), x_{n} \in E$ for each $n$ and such that $\left(\left\|x_{n}\right\|\right) \in \lambda$; the space $\lambda[E]$ is equipped with a natural norm topology given by $\|x\|_{x}=p\left[\left(\left\|x_{n}\right\|\right)\right]$.

2. $\lambda$-nuclear maps. Let $T$ be a linear map on the normed space $E$ into another, $F$. We define $T$ to be a $\lambda$-nuclear map if $T$ admits the representation

$$
T x=\sum_{n=1}^{\infty}\left\langle x, a_{n}\right\rangle y_{n}, \quad x \in E,
$$

where $a=\left(a_{n}\right) \in \lambda\left[E^{\prime}\right]$ and $y=\left(y_{n}\right) \in \lambda^{*}(F)$ with $\epsilon^{*}(y)<\infty$. There may be other representations of $T$ in the above form. Keeping this in mind, we define

$$
N_{\lambda}(T)=\inf \left\{\|a\|_{\pi^{*} \cdot \epsilon_{\lambda *}(y)}\right\}
$$

where the infimum is taken over all possible representations of $T$ in the above form.

We observe that $\lambda$-nuclear maps can be defined in the following equivalent way: say $T$ is $\lambda$-nuclear if $T$ has the representation

$$
T x=\sum_{n=1}^{\infty} \alpha_{n}\left\langle x, u_{n}\right\rangle y_{n},
$$

where $\left\|u_{n}\right\| \leqq 1$ for each $n, \alpha=\left(\alpha_{n}\right) \in \lambda$ and $y=\left(y_{n}\right) \in \lambda^{*}(F)$ with $\epsilon_{\lambda}^{*}(y) \leqq 1$. In this case

$$
N_{\lambda}(T)=\inf p(\alpha) .
$$

Let $N_{\lambda}(E, F)$ denote the set of all $\lambda$-nuclear maps on $E$ into $F$.

Theorem 1. Each $\lambda$-nuclear map $T$ is continuous and $\|T\| \leqq N_{\lambda}(T)$.

THEOREM 2. $N_{\lambda}(E, F)$ is a quasi-normed linear space under the norm $N_{\lambda}$; also if $F$ is a Banach space $N_{\lambda}(E, F)$ is complete if $\lambda$ is made of all sequences $u \in \omega$ for which $p(u)<\infty$.

THEOREM 3. If $A(E, F)$ denotes the space of all operators $T$ on $E$ which have finite dimensional ranges in $F$, then $A(E, F)$ is a dense subspace of $N_{\lambda}(E, F)$.

Corollary. If $F$ is a Banach space then each $T \in N_{\lambda}(E, F)$ is a compact linear map and each such $T$ has a separable range space.

The next two theorems play an important role in the factorization theorem (Theorem 6 ) characterizing $\lambda$-nuclear maps. 
THEOREM 4. Let $E, F$ and $G$ be normed linear spaces. Then we have the following:

(a) If $T \in N_{\lambda}(E, F)$ and $S \in L(F, G)$ then $S \circ T \in N_{\lambda}(E, G)$ and $N_{\lambda}(S \circ T) \leqq\|S\| \cdot N_{\lambda}(T)$.

(b) If $T \in L(E, F)$ and $S \in N_{\lambda}(F, G)$ then $S \circ T \in N_{\lambda}(E, G)$ and $N_{\lambda}(S \circ T) \leqq N_{\lambda}(S) \cdot\|T\|$.

Theorem 5. Let $\delta=\left(\delta_{n}\right)$ be a fixed member of $\lambda$. Then the map $D: l^{\infty} \rightarrow \lambda$ defined by $D(u)=\left(u_{i} \delta_{i}\right)$ is a $\lambda$-nuclear map and $N_{\lambda}(D)=p(\delta)$.

Theorem 6. Suppose $F$ is a Banach space. Then the map $T \in L(E, F)$ is $\lambda$-nuclear if and only if it can be factorized as follows:

$$
T=Q \circ D \circ P, \quad E \stackrel{P}{\rightarrow} \stackrel{D}{\rightarrow} \stackrel{Q}{\rightarrow} \lambda \stackrel{\rightarrow}{\rightarrow} F
$$

where $P$ and $Q$ are continuous linear maps with $\|P\| \leqq 1$ and $\|Q\| \leqq 1$ and $D$ is as defined in Theorem 5.

3. Quasi- $\lambda$-nuclear maps. A linear map $T$ on $E$ into $F$ is defined to be quasi- $\lambda$-nuclear if there exists a sequence $a=\left(a_{n}\right)$ of elements of $E^{\prime}$ such that $a \in \lambda\left[E^{\prime}\right]$ and $\|T x\| \leqq p\left[\left(\left|\left\langle x, a_{n}\right\rangle\right|\right)\right]$ for each $x \in E$. Set $Q_{\lambda}(T)=\inf \|a\|_{\pi}$, where the infimum is taken over all admissible $a$. Then one can prove that $Q_{\lambda}(E, F) \subset L(E, F)$ with $\|T\| \leqq Q_{\lambda}(T)$. Also $N_{\lambda}(E, F) \subset Q_{\lambda}(E, F)$ with $Q_{\lambda}(T) \leqq N_{\lambda}(T)$ for $T \in N_{\lambda}(E, F)$. In the opposite direction we have the following result.

THEOREM 7. If the Banach space $F$ has the extension property and if $T \in Q_{\lambda}(E, F)$ then $T \in N_{\lambda}(E, F)$ and $Q_{\lambda}(T)=N_{\lambda}(T)$.

We remark also that the above result is true for any pair $E, F$ provided the sequence space $\lambda$ is complemented. Thus for $\lambda=l^{2}$ when one gets the quasi-2-nuclear maps and the 2-nuclear maps, we have the (known) result that $N_{2}(E, F)=Q_{2}(E, F)$.

4. $\lambda$-nuclear maps and absolutely $\lambda$-summing maps. The linear map $T$ on $E$ into $F$ is said to be absolutely $\lambda$-summing if for each $x=\left(x_{n}\right) \in \lambda(E)$, the sequence $T x=\left(T x_{n}\right) \in \lambda[F]$. Let now $\lambda$ $=\{x \in \omega: p(x)<\infty\}$.

TheOREM 8. The linear map $T$ on $E$ into $F$ is absolutely $\lambda$-summing if and only if there exists a $\rho>0$ such that for each finite system of vectors $x_{1}, x_{2}, \cdots, x_{k}$ in $E$ the following inequality holds:

$\left\|\left(T x_{1}, T x_{2}, \cdots, T x_{k}, 0,0, \cdots\right)\right\|_{\pi} \leqq \rho \cdot \epsilon_{\lambda}\left(x_{1}, x_{2}, \cdots, x_{k}, 0,0, \cdots\right)$.

The smallest such $\rho$ is denoted $\pi_{\lambda}(T)$. It can be shown that when $F$ is a Banach space the space $\pi_{\lambda}(E, F)$ of all the absolutely $\lambda$-sum- 
ming maps on $E$ into $F$ is a Banach space with the norm defined by $\pi_{\lambda}(\cdot)$.

The space $\lambda$ is said to have the norm iteration property if for each sequence $\left(x^{n}\right)$ of elements of $\lambda$ we have $p\left[p\left(x^{n}\right)\right]=p\left[p\left(x_{i}\right)\right]$ where $x_{i}=\left(x_{i}^{1}, x_{i}^{2}, \cdots, x_{i}^{n}, \cdots\right)$. It is easily verified that the spaces $c_{0}$ and $l^{p}(1 \leqq p \leqq \infty)$ have the above property.

THEOREM 9. If $\lambda$ has the norm iteration property then $N_{\lambda}(E, F)$ $C \pi_{\lambda}(E, F)$ and $\pi_{\lambda}(T) \leqq N_{\lambda}(T)$.

We remark now that Theorem 9 above is true also for quasi- $\lambda$ nuclear maps with practically the same proof as that of Theorem 9 . In case $\lambda=l^{p}(p \geqq 1)$ the results of Persson and Pietsch [5] show that by taking the composition product of a certain finite number of $p$-quasi-nuclear maps one can obtain ultimately a nuclear map. In a rather general set up as ours we cannot prove a result of that type. Consequently when one attempts to formulate the concept of a $\lambda$-nuclear space using the standard canonical mappings, one obtains naturally two related concepts, those of $\lambda$-nuclear spaces and of quasi- $\lambda$-nuclear spaces.

\section{REFERENCES}

1. D. J. H. Garling, On symmetric sequence spaces, Proc. London Math. Soc. (3) 16 (1966), 85-106. MR 33 \#537.

2. - A class of reflexive symmetric BK-spaces, Canad. J. Math. 21 (1969), 602-608.

3. A. Grothendieck, Produits tensoriels topologiques et espaces nucleaires, Mem. Amer. Math. Soc. No. 16 (1955). MR 17, 763.

4. G. Köthe, Topologische lineare Räume. I, Die Grundlehren der math. Wissenschaften, Band 107, Springer-Verlag, Berlin and New York, 1960. MR 24 \#A411.

5. A. Persson and A. Pietsch, p-nukleare und p-integrale Abbildungen in Banachräumen, Studia Math. 33 (1969), 19-62.

6. A. Pietsch, Nukleare lokalkonvexe Räume, Akademie Verlag, Berlin, 1965. MR 31 \#6114.

7. W. Ruckle, Symmetric coordinate spaces and symmetric bases, Canad J. Math. 19 (1967), 828-838. MR 38 \#4977.

8. W. L. C. Sargent, Some sequence spaces related to the Ip spaces, J. London Math. Soc. 35 (1960), 161-171.

9. - On sectionally bounded BK-spaces, Math. Z. 83 (1964), 57-66. MR 28 \#2403.

10. K. Zeller, Theorie der Limitierungsverfahren, Ergebnisse der Mathematik und ihrer Grenzgebiete, Heft 15, Springer-Verlag, Berlin and New York, 1958. MR 22 \#9759.

University of Michigan, ANn ARbor, Michigan 48104

Goethe Universität, Frankfurt am Main 